\title{
Actuation and stiffening in fluid-driven soft robots using low-melting-point material*
}

\author{
Jan Peters ${ }^{1}$, Erin Nolan ${ }^{2}$, Mats Wiese ${ }^{1}$, Mark Miodownik ${ }^{2}$, Sarah Spurgeon ${ }^{3}$, Alberto Arezzo ${ }^{4}$ \\ Annika Raatz ${ }^{1}$, Helge A. Wurdemann ${ }^{2}$
}

\begin{abstract}
Soft material robots offer a number of advantages over traditional rigid robots in applications including humanrobot interaction, rehabilitation and surgery. These robots can navigate around obstacles, elongate, squeeze through narrow openings or be squeezed - and they are considered to be inherently safe. The ability to stiffen compliant soft actuators has been achieved by embedding various mechanisms that are generally decoupled from the actuation principle. Miniaturisation becomes challenging due to space limitations which can in turn result in diminution of stiffening effects. Here, we propose to hydraulically actuate soft manipulators with lowmelting-point material and, at the same time, be able to switch between a soft and stiff state. Instead of allocating an additional stiffening chamber within the soft robot, one chamber only is used for actuation and stiffening. Low Melting Point Alloy is integrated into the actuation chamber of a single-compartment soft robotic manipulator and the interfaced robotic syringe pump. Temperature change is enabled through embedded nichrome wires. Our experimental results show higher stiffness factors, from $9-12$ opposing the motion of curvature, than those previously found for jamming mechanisms incorporated in separate additional chambers, in the range of $2-8$ for the same motion.
\end{abstract}

\section{INTRODUCTION}

There has been an increased interest in soft material robotics research and the wider field of soft robotics, as seen in the development of dedicated journals such as "Soft Robotics", conferences such as the "IEEE International Conference on Soft Robotics", and sessions at major robotics conference such as the "IEEE International Conference on Robotics and Automation" and the "IEEE/RSJ International Conference on Intelligent Robots and Systems". Many of these new soft robotic systems are biomimetic or bioinspired, with researchers looking to natural systems to find solutions to some of the problems faced by traditional, rigid robotics [1]-[3]. Results demonstrate that soft material robots can outperform conventional robots in certain application areas [4], [5], [7], [20] such in Minimally Invasive Surgery

*This work is supported by the Springboard Award of the Academy of Medical Sciences (grant number: SBF003-1109) and the Engineering and Physical Sciences Research Council (grant numbers: EP/N509577/1, EP/R037795/1, and EP/S014039/1).

${ }^{1} \mathrm{~J}$. Peters, M. Wiese and A. Raatz are with the Institute of Assembly Technology, Leibniz University Hannover, Germany peters@match. uni-hannover. de

${ }^{2}$ E. Nolan, H.A. Wurdemann and M. Miodownik are with the Department of Mechanical Engineering, University College London, UK. h.wurdemanneucl.ac.uk

${ }^{3}$ S. Spurgeon is with the Department of Electronic Electrical Engineering, University College London, UK.

${ }^{4}$ A. Arezzo is with the Department of Surgical Sciences, University of Torino, Italy.

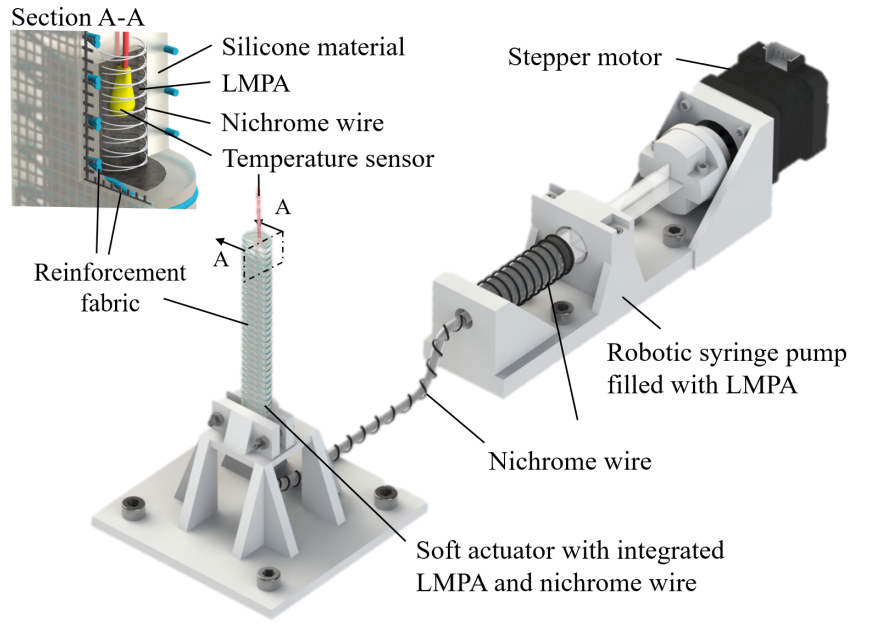

Fig. 1. Concept of the proposed soft, stiffness-controllable soft robotic manipulator actuated and stiffened using LMPA: The soft actuator is made of silicone with fabric-reinforced walls. The actuation chamber contains LMPA with an emdedded temperature transducer and integrated nichrome wire. The actuator is connected via a hose to the robotic syringe pump. Both parts are filled with LMPA which can be heated by nichrome wires.

(MIS), also known as laparoscopic or keyhole surgery [8][10]. In minimally invasive procedures, rigid laparoscopic instruments are inserted through $12-15 \mathrm{~mm}$ incisions, known as Trocar ports, which allow surgeons to carry out essential surgical interventions inside the patient's body [11]. During a number of MIS procedural steps, such as posterior and lateral Total Mesorectal Excision (TME), clinicians have reported challenges due to limited manoeuvrability of the rigid surgical tools [11], [12] an issue that soft robotics has shown great potential to address [11]. The benefits of soft manipulator structures include reduced abrasion of the internal soft tissue. The additional Degrees of Freedom (DoFs) provided by the soft structure allow for improved navigation around the organs inside the patient and thus avoid the need to cut through to reach the target [13], [14]. However the challenges of these soft structures include identifying methods of producing effective forces, and designing mechanisms to enable stiffness changes for soft Variable Stiffness Actuation (VSA) [15].

Several solutions to these complex challenges of soft stiffness variation have been investigated in recent years [31]. Some leverage opposing actuation principles to achieve stiffness, such as [16]-[20], whilst others explore mechanisms that vary the stiffness of an entire volume of material. 
In [21]-[23] for instance, granular jamming is integrated into an additional chamber in a silicone-based, pneumatically actuated soft manipulator. Stiffness variation can then be achieved by applying a vacuum to freeze the robots configuration in the desired position. Manipulators actuated by polymeric artificial muscles, as described in [24], were developed further in [25] to incorporate similar granule-filled chambers that used a vacuum to actuate and vary the stiffness of the manipulators joints. Instead of granules, some designs utilise layer jamming methods instead, such as the concept proposed in [27] where multiple overlapping layers of thin Mylar film are used to vary stiffness in a hollow, snake-like manipulator. Application of a vacuum increases the friction between the layers to generate a change in stiffness. Jamming principles significantly depend on the material choice, volume of material and negative pressure applied [26]. A VSA mechanism was reported in [28] that uses the heating and cooling of a wax-coated, open-cell foam to adjust the stiffness, strength, and volume of the structures. This method introduces time delays to the changes between soft and stiff states due to the delayed response of the material to the heating and cooling processes. Alternative materials, like Low Melting Point Alloys (LMPAs), are being explored to achieve similar effects, such as in [29], [30], In [30] for instance, additional chambers containing an LMPA were integrated into a surgical soft robotic manipulator.

Stiffening mechanisms are generally separated from the actuation mechanisms, using additional chambers and the stiffness control being decoupled from actuation chambers [31]. Miniaturisation becomes challenging due to space limitations which can in turn result in the diminution of stiffening effects [26], [32]. Current stiffening approaches also do not account for extreme changes in volume that can occur due the soft material robots distinct ability to deform, bend and elongate. Stiffening mechanisms should therefore be designed with the ability to adapt - introducing additional stiffening when the structures morph in shape and volume.

In this paper, we propose actuation chambers for fluidically driven soft manipulators that use LMPA to hydraulically actuate soft robots and, at the same time, switch between a soft and stiff state. Here, a bismuth-tin-indium composite is integrated into the actuation chamber of a singlecompartment soft manipulator and the interfaced robotic syringe pump. Temperature change within the LMPA is enabled through embedded nichrome wires. Below a temperature of $67^{\circ} \mathrm{C}$ the LMPA is solid and stiff but above this threshold the material is fluid and soft. Hence, it is possible to control the LMPA's, and therefore the manipulator's, stiffness. To compare our results with findings of existing jamming approaches, we based our design on a soft actuator presented in [33].

Section II gives an overview of our system including the design and manufacturing process of the LMPA-actuated soft robotic manipulator. In Section III, the interfaced robotic syringe pump and temperature control of the LMPA are described. The experimental protocol, results and a discussion are presented in Section IV. Section V lists the achievements of this paper and identifies future directions of our proposed simultaneous stiffening and actuation principle.

\section{CONCEPT AND FABRICATION OF AN LMPA-ACTUATED SOFT ROBOTIC MANIPULATOR}

\section{A. Concept and methodology}

The proposed system is shown in Figure 1. The two main components are a fluid-driven soft manipulator and the robotised syringe pump it is connected to via a hose. The entire volume of the syringe and hose is filled with an LMPA, and a nichrome wire covers the outside of both to enable heating of the actuation fluid.

The soft manipulator contains the LMPA with an embedded nichrome wire and temperature transducer (see section view A-A in Figure 1). The polymer of the manipulator is reinforced with two strain limiting components: a fibre wound around the outside; and an inextensible bottom layer. When the LMPA is melted, these strain limiting features transform the expansion due to pressurization into the bending motion of the actuator. The fibre prevents the LMPA chamber from expanding radially, resulting in a linear expansion instead, whilst the inextensible bottom layer prevents this expansion on the bottom side of the actuator. This causes the actuator to bend in a constant curvature around its bottom side.

The detailed design and fabrication process is presented in Section II-B.

\section{B. Fabrication of the LMPA-driven actuator}

Figure 2 shows the soft robotic actuator consisting of a $100 \mathrm{~mm}$ long and $13 \mathrm{~mm}$ wide silicone main body [Dragon Skin 10 Medium, SmoothOn] with a single chamber filled with the LMPA [Field's Metal, Hainess \& Maassen; 32.5\% bismuth, $16.5 \%$ tin, $51 \%$ indium]. The chamber of $85 \mathrm{~mm}$



(a)

(b)

(c)

Fig. 2. The silicone-based soft actuator based on the design in [33]: The overall length is $100 \mathrm{~mm}$ with a semi-circular cross-section of a $13 \mathrm{~mm}$ diameter. The planar surface is reinforced using non-extensible fabric. A fabric thread is helically twisted around the single compartment actuator which contains LMPA, an embedded temperature sensor and nichrome wire. 
length has a semi-circular cross-section (see section view A$A$ in Figure 2) with a diameter of $9 \mathrm{~mm}$ resulting in a volume of $2.7 \mathrm{~cm}^{3}$. There is a thermistor [NTC NI24 NA 0103, AVX] directly integrated into the LMPA at the actuator tip. The heating wire $[0.08 \mathrm{~mm}$ diameter trimel enamelled, Scientific Wire Company] is $1.80 \mathrm{~m}$ in length and curved in a helix inside the chamber. A polyester thread [0.2 mm, Korbond] is wound around the actuator and the flat bottom side of the actuator contains an inextensible layer [43T Polyester Mesh, Jackson's Art].

The fabrication method used was based on that used for other fluidic elastomer actuators (FEAs) with additions made for the integration of the LMPA as the actuation material. This process consisted of four steps: (1) the moulding of the silicone main body; (2) integration of the thermistor, resistance wire and the LMPA; (3) circumferential fibre windings along the chamber; (4) the attachment of an inextensible layer on the bottom. Initially, in Step (1), the two parts of the silicone were mixed in a 1:1 ratio, degassed and then poured into the 3D printed mold [made of Formlabs Clear Resin printed by a Formlabs Form $23 \mathrm{D}$ printer] to produce the main body. This part contains a hole on one side for tube insertion and remains open on the other side for the next stage of fabrication. For each silicone casting step like this, the mixture is left for approximately 24 hours to cure. In Step (2) the heating wire is wrapped around a $1.25 \mathrm{~mm}$ syringe needle to produce a double crossed helix. The two ends of the wire are then pulled through the hole left for tube insertion and fixed together with the tube and the main body using silicone glue [Sil-Proxy, Smooth-On]. The chamber and tube are then filled with the molten LMPA, and a thermistor then placed before solidification occurs. After solidification of the LMPA, the actuator is returned to the mold to add further silicone mixture to close the tip. The thermistor and the last winding of the heating wire are then enclosed by silicone to hold them in place. In Step (3) the polyester thread is wrapped around the actuator, first along the length and then again back towards the tube. The thread on the upper, round side of the actuator is wound straight across, perpendicular to the sides of the actuator, but once underneath it is wound diagonally across and up the bottom side with a pitch of $2 \mathrm{~mm}$. This configuration ensures good bending behaviour as the fibre only restricts the actuator expansion in the radial direction but not in the longitudinal. In Step (4), the polyester mesh is placed inside a mold, covered with a $2 \mathrm{~mm}$ layer of silicone mixture and degassed before the actuator main body is then placed on top. As the silicone sets, it binds the two parts together to create the final actuator.

\section{INTERFACING THE ROBOTIC SYRINGE PUMP WITH TEMPERATURE CONTROL FOR VISCOSITY CHANGE OF LMPA}

The overall integrated system consists of the LMPA-driven manipulator, a motorised syringe system and a temperature control unit as shown in Figure 3. A syringe is filled with the LMPA and connected via a tube to the manipulator. The thermistor placed inside the manipulator during fabrication is connected to a PID control setup to measure the temperature of the LMPA and adjust according to user input. The syringe and tube are permanently heated to ensure the actuation.

\section{A. Integration of the robotic (or motorized) syringe pump for LMPA-actuation}

A 3D printed base was adapted from [34] to hold the LMPA filled syringe [10 ml Omnifix Luer Lock, Braun] and barrel flange. The plunger flange is clamped in the $3 \mathrm{D}$ printed plunger clamp which is connected to the threaded shaft of the non-captive stepper motor [17HS5001-100D8 with anti-rotation mechanism, RobotDigg, Shanghai, China]. A heating wire $[0.6 \mathrm{~mm}$ constantan resistance wire, Block] is wrapped around the syringe and tube, with approximately $1 \mathrm{~mm}$ and $3 \mathrm{~mm}$ helical pitches respectively, to keep the LMPA inside in a liquid state. The stepper motor is connected to an Arduino [ATmega328P Arduino Uno, Arduino] with a motorshield [KA03, Velleman Kit]. The stepper motor, and thereby the syringe, is controlled by the user over a graphical user interface (GUI), also adapted from [34] that was built with the programming language Processing. A standard Firmata firmware is used to enable communication between the Arduino and the GUI. This allows the syringe direction, volume displacement and the stepper motor speed to be controlled via user input in the GUI.

\section{B. Implementation of a PID temperature control}

The temperature of the LMPA inside the actuator is controlled by a PID control system implemented using the Arduino. The thermistor embedded in the manipulator is connected to the Arduino so the temperature can be calculated from the resistances measured. A PID program written using the standard Arduino PID control library, the parameters optimised using the Ziegler-Nichols method, was then used to calculate the necessary pulse width modulation needed to control the current through the heating wires with

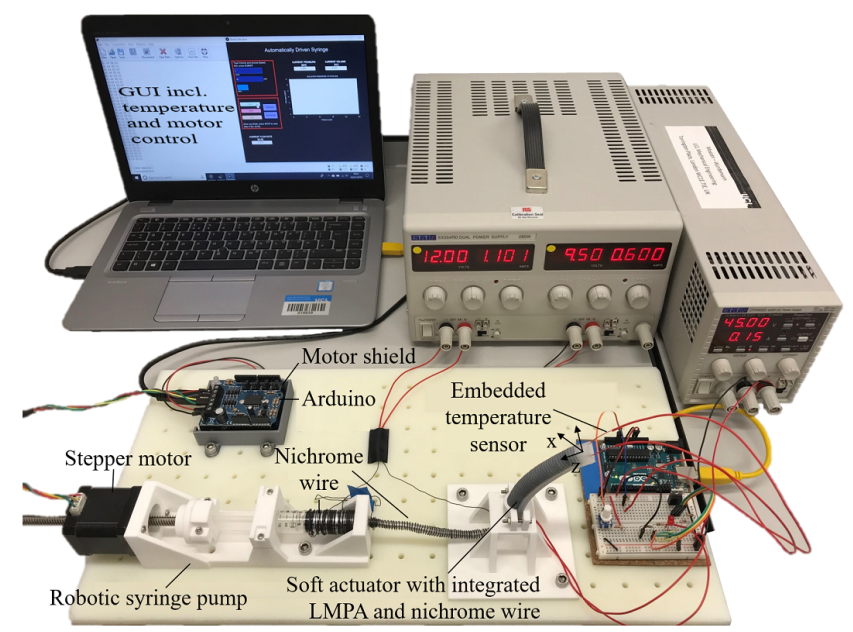

Fig. 3. The overall setup consists of a robotic syringe pump connected to a single compartment soft robotic actuator. Together with the nichrome wire, a temperature transducer is embedded into the soft actuator. The stepper motor of the syringe pump and temperature control unit is interfaced through an Arduino with a GUI. 


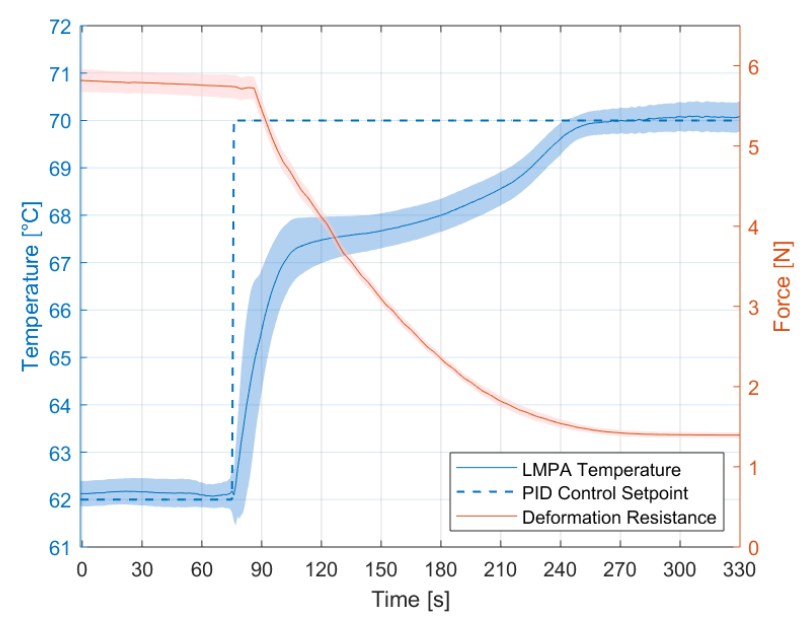

Fig. 4. Stiffness response (solid red line) of the soft actuator when the PID temperature controller (solid blue line) is excited by a step input (dotted blue line) from $62^{\circ} \mathrm{C}$ to $70^{\circ} \mathrm{C}$. The tip of the actuator is displaced by a force/torque sensor by $7 \mathrm{~mm}$ when in solid state at $62^{\circ} \mathrm{C}$. The force decreases when the temperature increases and the LMPA liquefies.

a logic level MOSFET [FQP30N06L, ON Semiconductor]. The setpoint of the PID temperature control can be changed by the user with a potentiometer.

Figure 4 shows the liquefying process of the actuator and time response of the temperature control. The actuator tip is repeatedly displaced at a speed of $6.25 \frac{\mathrm{mm}}{\mathrm{s}}$ by $7 \mathrm{~mm}$ at $62^{\circ} \mathrm{C}$. The force is measured at the maximal deflection after a $0.1 \mathrm{~s}$ stop. At the same time the temperature of the LMPA is measured. Changing the setpoint of the temperature control to $70^{\circ} \mathrm{C}$ leads to a decreasing resistance force of the actuator. The system needs 190 s to switch between $62^{\circ} \mathrm{C}$ and $70^{\circ} \mathrm{C}$ causing a force drop of $4.8 \mathrm{~N}$. The reverse process resulting in the LMPA to stiffen requires about $300 \mathrm{~s}$.

\section{EXPERIMENTAL PROTOCOL, RESULTS AND DISCUSSION}

\section{A. Test Protocol}

To characterise the LMPA-actuated manipulator fabricated and interfaced in Figure 3, three experimental tests have been carried out:

Experiment 1: The tip of the soft manipulator is deflected in its default configuration by $7 \mathrm{~mm}$ in $x$ - and $y$ direction when the LMPA is liquefied at $70^{\circ} \mathrm{C}$ and solidified at $62^{\circ} \mathrm{C}$. Recording the forces during the tip displacement will explore the stiffness of the actuator.

Experiment 2: The soft manipulator is actuated using $\Delta 1.9 \mathrm{ml}$ LMPA until it achieves $90^{\circ}$ bending. Again, the tip is deflected by $7 \mathrm{~mm}$ in $x$-, $y$ - and $z$-direction when the LMPA is liquefied at $70^{\circ} \mathrm{C}$ and solidified at $62^{\circ} \mathrm{C}$. Analysing the forces during the deflection will investigate the stiffness of the soft manipulator.

Experiment 3: The soft robot is gradually actuated by LMPA using the robotic syringe pump using $\Delta 3 \mathrm{ml}$. The location of the manipulator's tip is recorded.
To conduct Experiments 1 and 2, a test rig was set up comprising a robotised linear rail [Zaber X-LSM100A] equipped with a 6-axis force/torque (F/T) sensor [IIT-FT17]. A customised 3D printed indenter is mounted on the F/T sensor to accurately penetrate the tip of the soft actuator when the linear displaces the sensor by $7 \mathrm{~mm}$ at $0.21 \frac{\mathrm{mm}}{\mathrm{s}}$. The linear rail and F/T sensor are integrated via a MATLAB interface.

\section{B. Experimental results}

The displacement-force results of Experiments 1 and 2 are plotted in Figures 5 and 6. Insets show how the F/T sensor is placed and exerts forces to the manipulator's tip. Each graph shows the mean value and standard deviation of five repetitions. Blue solid lines represent stiffness curves of solidified LMPA, red dashed lines stiffness results of liquefied LMPA during loading and unloading cycles. In Figures 5(a) and (b), the forces $F_{x}$ and $F_{y}$ are collected when the manipulator's tip is displaced. When the LMPA is liquefied, the maximum force at a $7 \mathrm{~mm}$ deflection is $0.1 \mathrm{~N}$ in $F_{x}$ and $0.4 \mathrm{~N}$ in $F_{y}$, respectively. The hysteresis is

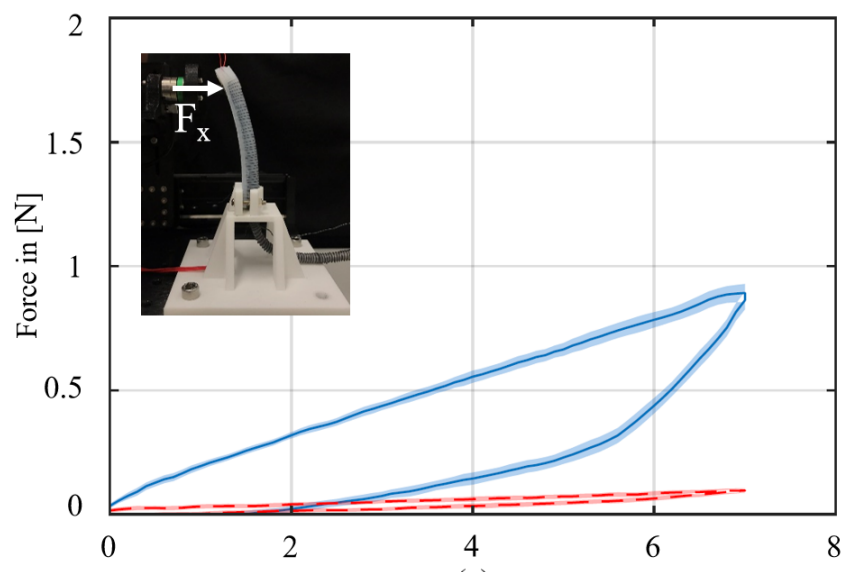

(a)

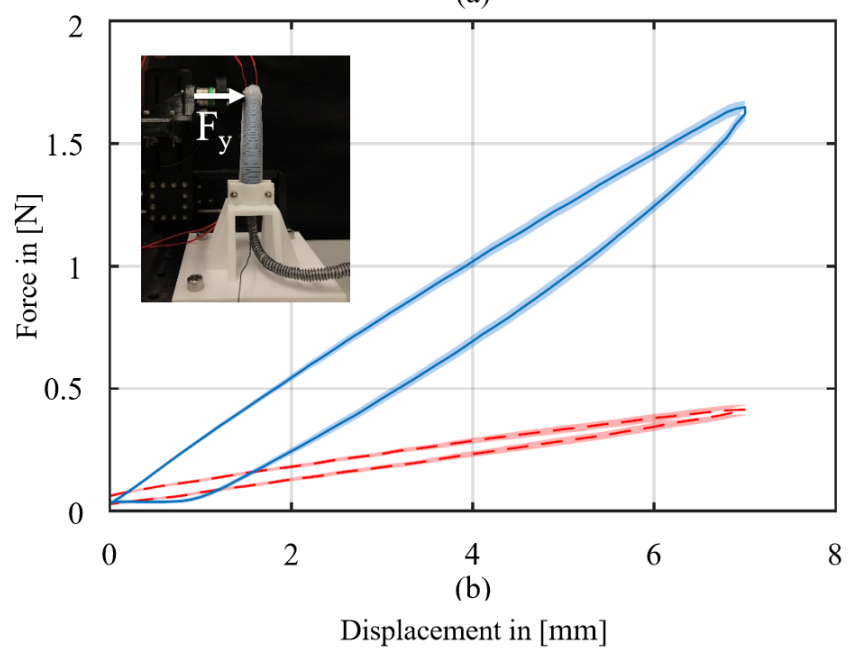

Fig. 5. Results of Experiment 1: The tip of the soft manipulator is deflected in its default configuration by $7 \mathrm{~mm}$ in (a) $x$ - and (b) $y$-direction when the LMPA is liquefied at $70^{\circ} \mathrm{C}$ (red dashed curve) and solidified at $62^{\circ} \mathrm{C}$ (blue solid curve). 

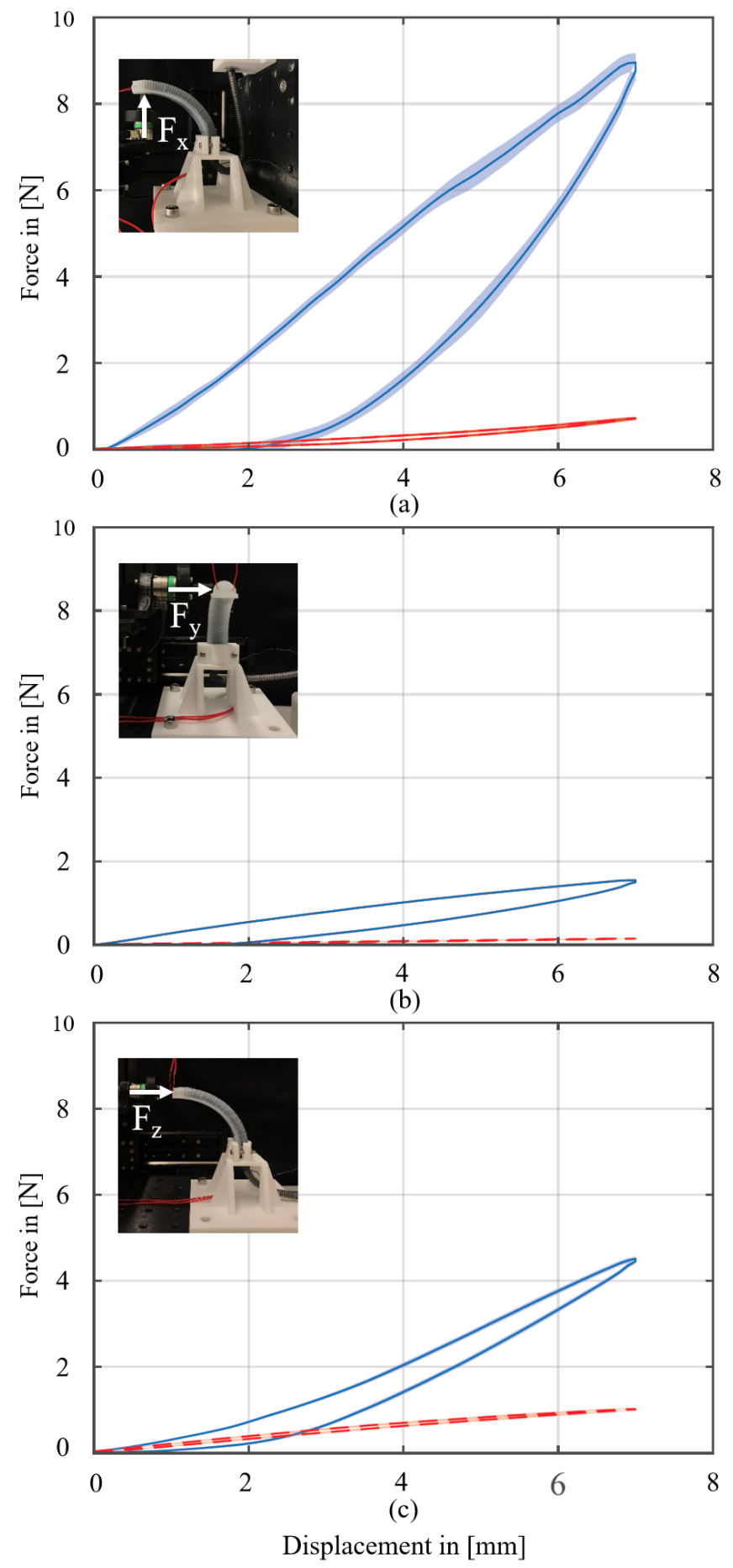

Fig. 6. Results of Experiment 2: The soft manipulator is actuated using $1.9 \mathrm{ml}$ LMPA until it bends $90^{\circ}$. The tip is deflected by $7 \mathrm{~mm}$ in (a) $x$-, (b) $y$ - and (c) $z$-direction when the LMPA is liquefied at $70^{\circ} \mathrm{C}$ (red dashed curve) and solidified at $62^{\circ} \mathrm{C}$ (blue solid curve).

negligible. When the LMPA is solid, the measured forces at a $7 \mathrm{~mm}$ deflection are $F_{x}=0.9 \mathrm{~N}$ and $F_{y}=1.6 \mathrm{~N}$, respectively. Whereas the loading curves shows a linear behaviour, the unloading curves form a hysteresis loop of $63 \%$ and $28 \%$. It is important to note that the unloading curve decreases its slope at about $5.5 \mathrm{~mm}$ in $x$ - and $1 \mathrm{~mm}$ in $y$-direction.
In Figure 6, the forces $F_{x}, F_{y}$ and $F_{z}$ are plotted when the manipulator's tip is displaced by $7 \mathrm{~mm}$ at $90^{\circ}$ bending. When the LMPA inside the soft actuator is above a temperature of $67^{\circ} \mathrm{C}$ and the material is fluid, the loading and unloading curve in all directions is almost linear showing a negligible amount of hysteresis similar to the results of Experiment 1 in Figure 5. The maximum forces at a $7 \mathrm{~mm}$ deflection are $0.9 \mathrm{~N}$ in $F_{x}, 0.1 \mathrm{~N}$ in $F_{y}$ and $1 \mathrm{~N}$ in $F_{z}$. When the LMPA temperature is below $67^{\circ} \mathrm{C}$ and the material is solid, forces of $9 \mathrm{~N}$ in $F_{x}, 1.7 \mathrm{~N}$ in $F_{y}$ and $4.5 \mathrm{~N}$ in $F_{z}$. While the loading curves can be approximated using a linear function, the unloading curves are nonlinear. In fact, the slope of the unloading curve suddenly decreases in the interval of $2-3 \mathrm{~mm}$. The hysteresis is largest in $x$-direction $(51 \%)$. In $y$ - and $z$-direction, the hysteresis is $46 \%$ and $24 \%$, respectively.

Figure 7 shows the results of Experiment 3. The position of the soft manipulator's tip is recorded when actuated using $\Delta[1,2,3] \mathrm{ml}$ LMPA. The experiment was repeated five times. The standard deviation was low, 0.66 in the $x$-direction and 0.88 in the $y$-direction, suggesting a high repeatability of these results.

\section{Discussion}

The displacement-force graphs of Figures 5 and 6 show a larger hysteresis than for those with the liquid LMPA when the LMPA inside the actuator is solid, but also a rapid decrease in slope, for instance at $5.5 \mathrm{~mm}$ in Figure 5(a), on the unloading curves for the solid LMPA. The displacement by the linear rail and $\mathrm{F} / \mathrm{T}$ sensor cause a plastic rather than elastic deformation when the LMPA is solid. The elastic

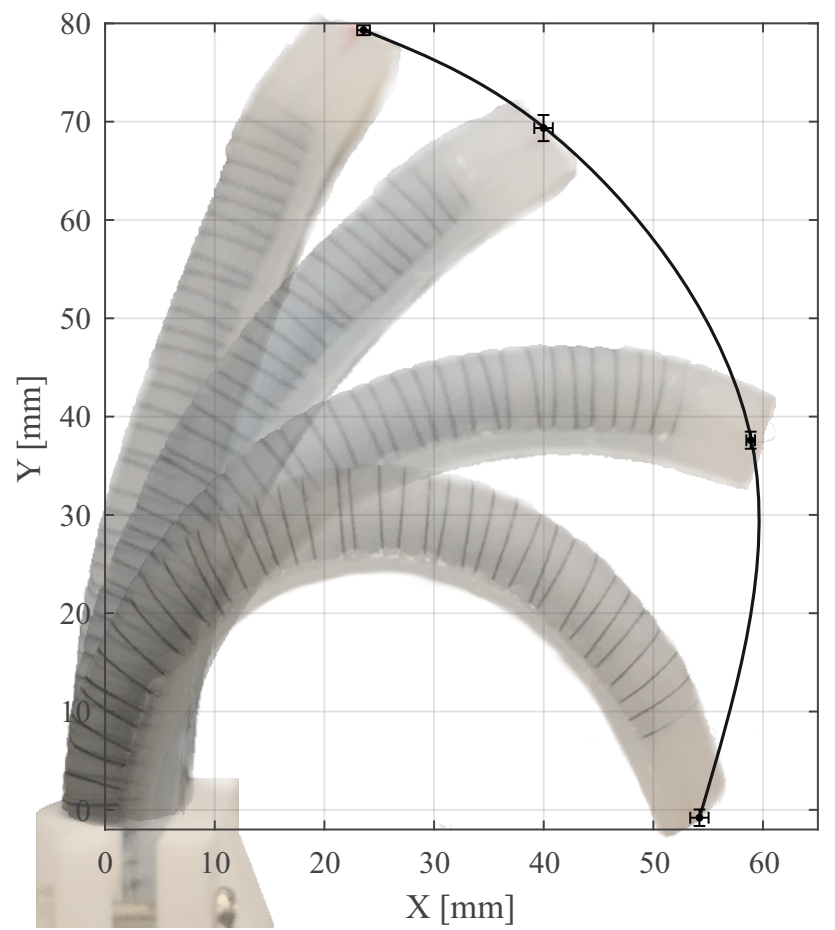

Fig. 7. Results of Experiment 3: The soft manipulator is fluidically actuated in incremental steps of $\Delta 1 \mathrm{ml}$. The position is recorded for five repetitions. 
component - see the initial unloading curve - is introduced by the silicone body of the soft actuator. The effect fades when the slope of the unloading curve rapidly decreases the manipulator does not return to its initial position.

Higher forces are achieved for the solid state across all results of Experiment 2 (see Figures 6) compared to Experiment 1 (see Figures 5) though the manipulator elongates during actuation. The novelty of our proposed concept is the ability to add LMPA (stiffening material) into the fluidic chamber which increases in volume when actuated (as the case in Experiment 2) - more stiffening material results in higher stiffness. It is also important to mention that the distance between the manipulator base and tip (which can be considered as the mathematical lever arm) is decreased in length when the manipulator bends by $90^{\circ}$ compared the default position in Experiment 1. Looking closer at Figures 5(a) and (b), the maximum forces that can be achieved with solid and liquefied LMPA state are smaller in $x$ - than in $y$-direction despite a larger moment of inertia in $x$ direction. As illustrated in Figure 2, a layer of reinforcement fabric is integrated on the planar side using an additional $2 \mathrm{~mm}$ thick layer of silicone material. This elastic buffer needs to be overcome before the actual stiffness of the LMPA is effective. A closer look at Figures 6(a) - (c) reveals that the highest force values can be achieved when the tip is displaced by $7 \mathrm{~mm}$ in $x$-direction. A displacement in $y$ direction results with the smallest amount of force. When the manipulator tip is displaced in $x$ - and $z$-direction in a $90^{\circ}$ configuration, the manipulator acts as a lever arm resulting in compressing and buckling LMPA structure. Less effect is noticeable in $z$-direction due to the thickness of the silicone at the manipulator's tip. In $y$-direction however, the buckling effect does not occur.

The design of our soft actuator was chosen so that a comparison to the jamming principles in [33] can be concluded. The ratio between the solid and liquefied LMPA state of the manipulator is determined. The bending stiffness is described by ratio of applied forces and the deflection $w$ (here constant) of the actuator $K=\frac{F}{w}$. The stiffening factor $s$ yields $\frac{F_{\text {solid }}}{F_{\text {liquid }}}$. Table I compares jamming methods in [33] with our approach reporting on the volume of the cavity in which the stiffening mechanism is embedded as well as the stiffening factors in $x$ - and in $y$-direction. It can be concluded that the stiffening factors are larger when using LMPA compared to jamming mechanisms. Looking into the experimental results

TABLE I

COMPARISON OF VARIOUS STIFFENING MECHANISMS: JAMMING APPROACHES [33] VERSUS THE PROPOSED STIFFENING CONCEPT

\begin{tabular}{c|c|c|c} 
& $\begin{array}{c}\text { Granular } \\
\text { jamming }\end{array}$ & $\begin{array}{c}\text { Layer } \\
\text { jamming }\end{array}$ & LMPA \\
\hline \hline Stiffening volume in $\left[\mathrm{cm}^{3}\right]$ & $7-22$ & $14-20$ & $2.7-3.6$ \\
\hline Stiffening factor in $x$-direction & $2-4$ & $2-8$ & $9-12$ \\
\hline Stiffening factor in $y$-direction & 2 & $2-4$ & $4-10$
\end{tabular}

of [33] in more detail, both jamming approaches return a lower stiffening factor when the manipulator is actuated and not in the default configuration. With our presented approach however, the stiffening factor becomes larger as LMPA is the actuation means and additional material is added into actuation chamber increasing the absolute stiffness and the stiffness factor.

\section{CONCLUSIONS}

We presented, for the first time, a fluidic actuation system that is able to shift stiffness-controllable material (LMPA, here a bismuth-tin-indium composite) in and out of an actuation chamber of a soft robotic manipulator. In this paper, we described our entire system: the robotic syringe pump and the design of the soft actuator inspired by [33] with integrated temperature sensor, nichrome wire and LMPA. The viscosity and stiffness of the actuation material can be changed through the temperature control of the embedded nichrome wire. Experimental results show an improved effectiveness of the stiffening mechanism compared to other previous designs. This is shown in the higher stiffness factors achieved, both opposing and perpendicular to the curvature of the actuator, than those of jamming mechanisms contained in separate, additional chambers. Yet the use of a single chamber design also improves the potential for miniaturisation and adaptability to the environment, allowing the volumes of LMPA to vary as the robot elongates or bends.

Temperature control is challenging. Liquefying and solidifying LMPA is time-consuming and depends on the amount of integrated nichrome wires, the volume of the LMPA and outside temperature. In a different way from jamming methods, allowing variable stiffness, LMPA has two states: liquid or solid. In future works, we will explore multiple distributed actuation chambers with smaller volumes. Our aim is to decrease the time to control the stiffness of the LMPA and enable variable stiffness by activating a combination of chambers.

\section{REFERENCES}

[1] I.D. Walker, D.M. Dawson, T. Flash, F.W. Grasso, R.T. Hanlon, B. Hochner, W.M. Kier, C.C. Pagano, C.D. Rahn, Q.M. Zhang, "Continuum robot arms inspired by cephalopods", UGVT VII, 2005.

[2] C. Laschi, B. Mazzolai, V. Mattoli, M. Cianchetti, P. Dario, "Design of a biomimetic robotic octopus arm”, Bioinspiration and Biomimetics, vol 4(1), pp. 1-8, 2009.

[3] M. Cianchetti, A. Arienti, M. Follador, B. Mazzolai, P. Dario, C. Laschi, "Design concept and validation of a robotic arm inspired by the octopus", Materials Science and Engineering C, vol 31(6), pp. 1230-1239, 2011.

[4] A. Stilli, H.A. Wurdemann, K. Althoefer, "A Novel Concept for Safe, Stiffness-Controllable Robot Links", Soft Robotics, vol. 4(1), pp. 1622, 2016

[5] A. Stilli, L. Grattarola, H. Feldmann, H.A. Wurdemann, K. Althoefer, "Variable Stiffness Links VSL Toward Inherently Safe Robotic Manipulators", in IEEE International Conference on Robotics and Automation, pp. 4971-4976, 2017.

[6] A. Stilli, A. Cremoni, M. Bianchi, A. Ridolfi, F. Gerli, F. Vannetti, H.A. Wurdemann, B. Allotta, K. Althoefer, "AirExGlove A Novel Pneumatic Exoskeleton Glove for Adaptive Hand Rehabilitation in Post-Stroke Patients", IEEE International Conference on Soft Robotics, pp. 579-584, 2018. 
[7] A. Palombi, G.M. Bosi, S. Di Giuseppe, E. De Momi, S. HomerVanniasinkam, G. Burriesci, H.A. Wurdemann, "Sizing the aortic annulus with a robotised, commercially available soft balloon catheter: in vitro study on idealised phantoms", in IEEE International Conference on Robotics and Automation, 2019.

[8] M. Cianchetti, T. Ranzani, G. Gerboni, I. de Falco, C. Laschi, A. Menciassi, "STIFF-FLOP Surgical Manipulator: Mechanical Design and Experimental Charaterization of the Single Module", IEEE/RSJ International Conference on Intelligent Robots and Systems, pp. 35763581, 2013.

[9] J. Fras, J. Czarnowski, M. Macias, J. Glowka, M. Cianchetti, A. Menciassi, "New STIFF-FLOP module construction idea for improved actuation and sensing", IEEE International Conference on Robotics and Automation, pp. 2901-2906, 2015.

[10] A. Shiva, H. Sadati, Y. Noh, J. Fras, A. Ataka, H. Wurdemann, H. Hauser, I. Walker, T. Nanayakkara, K. Althoefer, ”Elasticity vs. Hyperelasticity Considerations in Quasi-Static Modelling of a Soft Fingerlike Robotic Appendage for Realtime Position Force Estimation", Soft Robotics, vol. 6 (2), 2019.

[11] M. Cianchetti, T. Ranzani, G. Gerboni, T. Nanayakkara, K. Althoefer, P. Dasgupta, A. Menciassi, "Soft Robotics Technologies to Address Shortcomings in Today's Minimally Invasive Surgery: The STIFFFLOP Approach", Soft Robotics, vol. 1(2), pp. 122-131, 2014.

[12] H. Yamashita, A. Iimura, E. Aoki, T. Suzuki, T. Nakazawa, E. Kobayashi, M. Hashizume, I. Sakuma, T. Doh, "Development of endoscopic forceps manipulator using multi-slider linkage mechanisms," Asian Symposium on Computer Aided Surgery - Robotic and Imageguided Surgery, 2005.

[13] A. Arezzo, Y. Mintz, M.E. Allaix, S. Arolfo, M. Bonino, G. Gerboni, M. Brancadoro, M. Cianchetti, A. Menciassi, H.A. Wurdemann, Y. Noh, K. Althoefer, J. Fras, J. Glowka, Z. Nawrat, G. Cassidy, R. Walker, M. Morino, Total mesorectal excision using a soft and flexible robotic arm: a feasibility study in cadaver models, Surgical Endoscopy, vol. 31(1), pp. 264273, 2016.

[14] A. Althoefer, H.A. Wurdemann, A. Shafti, A. Shiva, J. Konstantinova, "Soft and Stiffness-controllable Robotics Solutions for Minimally Invasive Surgery The STIFF-FLOP Approach", River Publishers Series in Automation, Control and Robotics, April 2018.

[15] C. Laschi, B. Mazzolai, M. Cianchetti, "Soft robotics: Technologies and systems pushing the boundaries of robot abilities", Science Robotics, vol. 1(1), pp. 3690, 2016.

[16] A. Stilli, H.A. Wurdemann, K. Althoefer, "Shrinkable, stiffnesscontrollable soft manipulator based on a bio-inspired antagonistic actuation principle", in IEEE/RSJ International Conference on Intelligent Robots and Systems, Chicago, USA, pp. 2476-2481, 2014.

[17] F. Maghooa, A. Stilli, K. Althoefer, H.A. Wurdemann, "Tendon and pressure actuation for a bio-inspired manipulator based on an antagonistic principle", IEEE International Conference on Robotics and Automation, pp. 2556-2561, 2015.

[18] H.A. Wurdemann, A. Stilli, K. Althoefer, "An antagonistic actuation technique for simultaneous stiffness and position control", in Lecture Notes in Computer Science: An Antagonistic Actuation Technique for Simultaneous Stiffness and Position Control, Springer, pp. 164-174, 2015.

[19] A. Shiva, A. Stilli, Y. Noh, A. Faragasso, K. Althoefer and H.A. Wurdemann, "Tendon-based stiffening for a pneumatically actuated soft manipulator", IEEE Robotics and Automation Letters, vol. 1(2), pp. 632-637, 2016.

[20] A. Stilli, K. Althoefer, H.A. Wurdemann, "Soft Robotics. Bio-inspired Antagonistic Stiffening", Developing Support Technologies, Biosystems Biorobotics, Springer, pp. 207-214, 2018.

[21] A. Jiang, E.L. Secco, H. Wurdemann, T. Nanayakkara, P. Dasgupta, K. Athoefer, "Stiffness-controllable octopus-like robot arm for minimally invasive surgery", Workshop on New Technologies for Computer/Robot Assisted Surgery, 2013.

[22] M. Li, T. Ranzani, S. Sareh, L.D. Seneviratne, P. Dasgupta, H.A. Wurdemann, K. Althoefer, "Multi-fingered haptic palpation utilising granular jamming stiffness feedback actuators", Smart Materials And Structures, vol. 23(9), pp. 1-11, 2014.

[23] T. Ranzani, G. Gerboni, M. Cianchetti, A. Menciassi, "A bioinspired soft manipulator for minimally invasive surgery", Bioinspiration \& Biomimetics, vol. 10(3), pp. 035008, 2015.

[24] D.G. Caldwell, G.A. Medrano-Cerda, M. Goodwin, "Control of pneumatic muscle actuators", IEEE Control Systems, vol. 15, 1995.
[25] A. Jiang, G. Xynogalas, P. Dasgupta, K. Althoefer, T. Nanayakkara, "Design of a variable stiffness flexible manipulator with composite granular jamming and membrane coupling", IEEE/RSJ International Conference on Intelligent Robots and Systems, pp. 2922-2927, 2012.

[26] A. Jiang, P. Agrawal, K. Althoefer, T. Nanayakkara, P. Dasgupta, "Bioinspired connective granular jamming for a robotic limb," Transactions of Japanese Society for Medical and Biological Engineering, vol.51, pp.R-270R-270, 2013.

[27] Y.J. Kim, S. Cheng, S. Kim, K. Iagnemma, "Design of a Tubular Snake-like Manipulator with Stiffening Capability by Layer Jamming", IEEE/RSJ International Conference on Intelligent Robots and Systems, pp. 4251-4256, 2012.

[28] N.G. Cheng, A. Gopinath, L. Wang, K. Iagnemma, A.E. Hosoi, "Thermally Tunable, Self-Healing Composites for Soft Robotic Applications", Macromolecular Materials and Engineering, vol. 299(11), pp. 1279-1284, 2014.

[29] J. Shintake, B. Schubert, S. Rosset, H. Shea, D. Floreano, "Variable stiffness actuator for soft robotics using dielectric elastomer and lowmelting-point alloy," IEEE/RSJ International Conference on Intelligent Robots and Systems, pp. 1097-1102, 2015.

[30] H. Abidi, A. Tonazzini, M. Cianchetti, D. Floreano, A. Menciassi, "Low Melting Point Alloy Based Stiffening of a Soft Robot", International Congress of the Society for Medical Innovation and Technology, 2017.

[31] M. Manti, V. Cacucciolo, M. Cianchetti, "Stiffening in Soft Robotics: A Review of the State of the Art," IEEE Robotics and Automation Magazine, vol. 23(3), pp. 93-106, 2016.

[32] H. Abidi, M. Brancadoro, A. Diodato, M. Cianchetti, H.A. Wurdemann, K. Althoefer, A. Menciassi, "Highly Dexterous 2-module Soft Robot for Intra-organ Navigation in Minimally Invasive Surgery", The International Journal of Medical Robotics and Computer Assisted Surgery, vol. 14 (1), pp. e1875, 2018.

[33] V. Wall, R. Deimel, O. Brock, "Selective stiffening of soft actuators based on jamming", IEEE International Conference on Robotics and Automation, pp. 252-257, 2015.

[34] S. Di Guiseppe, "Transcatheter aortic valve replacement : measuring physiological compliance and vessel geometry using a valvuloplasty balloon catheter", M.S. Thesis, Politecnico Di Milano, Milan, Italy, 2017. Retrieved from http://hdl.handle.net/10589/138100. 